\title{
Medical Imaging Scans: A Short Letter
}

\section{Dogra A $^{1 *}$, Goyal B ${ }^{1}$, Agrawal $\mathbf{S}^{1}$ and Sohi BS ${ }^{2}$}

1UIET, Panjab University, Chandigarh, India

${ }^{2}$ Chandigarh University, Chandigarh, India

*Corresponding author: Ayush Dogra, UIET, Panjab University, Chandigarh, India, E mail: ayush123456789@gmail.com

\section{Short communication}

Volume 2 Issue 1

Received Date: February 06, 2018

Published Date: February 16, 2018

\section{Abstract}

The medical imaging scans of various organs of the body have become an indispensible tool for medical fraternity which helps them in planning various surgical procedures. These scans depict the anatomical, structural and functional molecular level information of the subject under study. In this letter a brief description has been given for the same.

Keywords: Medical scans; Computed tomography; X-ray; Imaging; Denoise; Fusion

Abbrevations: CT: Computed Tomography; MRI: Magnetic Resonance Imaging; PET: Positron Emission Tomography; SPECT: Single Photon Emission Tomography; DSA: Digital Subtraction Angiography.

\section{Short Communication}

The various abnormalities in the human body are made visible by these medical scans on a radiographic plane. These X-ray scans helps in the diagnosis, treatment and staging of various ailments in the human body. The basic physics behind the comprehension of medical imaging lies in elementary nuclear physics and the nature of electromagnetic radiation [1].

The various types of medical imaging technologies are CT Computed Tomography), (MRI (Magnetic Resonance Imaging), PET (Positron Emission Tomography), X-Ray, Ultrasound, SPECT (Single Photon Emission Tomography) and DSA (Digital Subtraction Angiography). These imaging modalities finds applications in assessment of various organs of the body like brain, bone, teeth, soft tissues, blood vessels, breast and stomach etc [2].

These images are obtained from multiple sensors. For instance a CT and MRI images can be obtained for the similar view of the subject under study. These multi- sensor imaging modalities are fused together in order to enable the visualization of the complimentary information [3]. Sometimes these images are corrupted with sensor noise i.e. Gaussian noise. Therefore these scans have to denoised in order to make precise and accurate diagnosis [4]. In X-ray scans the X-ray are blocked $y$ the high density soft tissues and bones which make visible on a radiographic plan. X-ray finds application in the identification of ailments like pneumonia, fractures and dental cavities. This is one of the most basic radiography technologies under application.

On the hand CT scans enable to visualization of those areas which have contrast variation less than 5\%.CT scans are also employed in diagnosis and staging of cancer and abdominal diseases. Another class of medical imaging is Emission Computed Tomography which helps in understanding the basic physiology of the organs as well as functions and mapping associated with those organs. The two types of emission computed tomography are PET (Positron Emission Tomography) SPECT (Single Positron Emission Computed Tomography).PET and SPECT are the two research technologies which extremely important and extensive application in medical oncology: cancer detection, staging and treatment and management [1]. 


\section{Open Access Journal of Pharmaceutical Research}

Digital subtraction angiography (DSA) is a modern fluoroscopic radiographic technology employed to diagnose cardio-vascular diseases. The DSA images are obtained by injecting the fluoroscopic agent in the human body. This technology finds applications in monitoring the blood flow in the various vessel organs of the human body [5]. In MRI the organ under study is subjected to strong magnetic field. This technology helps in patient management and treatment without exposing the patients to ionizing radiations Ultrasound is the sound wave which is not audible to the human ear with frequency ranging between $20 \mathrm{Khz}$ to gigahertz. Ultrasound imaging generates the high amplitude temporal frequencies which help in real time monitoring of the fetus in womb. The Figure 1 given below gives an illustration of each of the various radiographic scans discussed above.

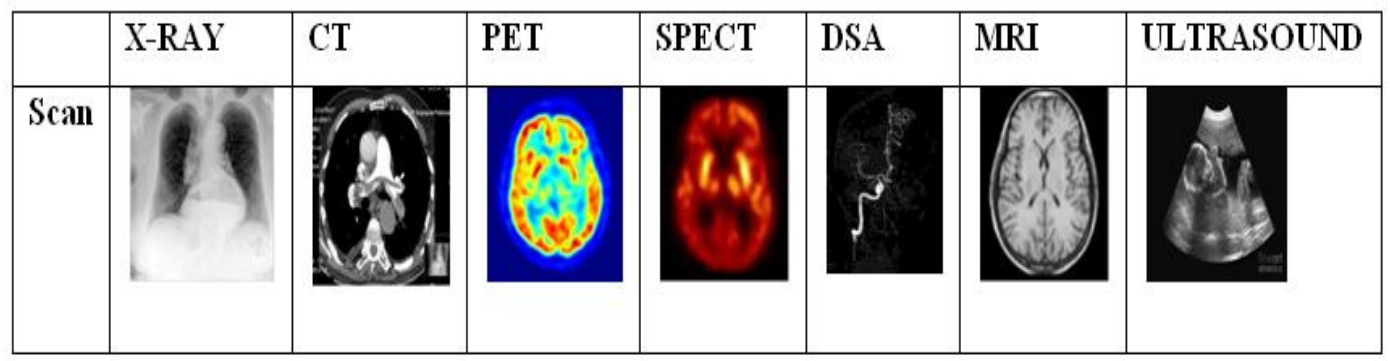

Figure1: An example of the each of the various radiographic techniques.

Therefore for the above briefing that each of the radiographic scans has its own function and application. For the extensive and complete treatment planning of a specific ailment one may different types of radiographic scans. These scans are essential for precise and accurate diagnosis. In the field of research and development, while developing various image denoising and image fusion schemes one needs an elementary knowledge of the principle working, contrast levels and display and applications of the various imaging modalities. The radiography departments are required to maintain strict health measurements pertaining of the harmful ionizing and fluoroscopic adverse effects of these technologies.

\section{References}

1. Dogra Ayush, Bhawna Goyal, Sunil Agrawal (2017) From multi-scale decomposition to non-multi-scale decomposition methods: A comprehensive survey of image fusion techniques and its applications. IEEE Access 5: 16040-16067.
2. James AP, Dasarathy BV (2014) Medical image fusion: A survey of the state of the art. Information Fusion 19: 4-19.

3. Dogra A, Goyal B, Agrawal S (2018) Osseous and digital subtraction angiography image fusion via various enhancement schemes and Laplacian pyramid transformations. Future Generation Computer Systems.

4. Goyal B, Dogra A, Agrawal S, Sohi BS (2018) Twodimensional gray scale image denoising via morphological operations in NSST domain \& bitonic filtering. Future Generation Computer Systems.

5. Dogra A, Goyal B, Agrawal S, Ahuja CK ( 2017) Efficient fusion of osseous and vascular details in wavelet domain. Pattern Recognition Letters 94: 189193. 